\title{
Disruption of the CXCR4/CXCL12 chemotactic interaction during hematopoietic stem cell mobilization induced by GCSF or cyclophosphamide
}

\author{
Jean-Pierre Lévesque, ${ }^{1}$ Jean Hendy, ${ }^{1}$ Yasushi Takamatsu, ${ }^{2}$ Paul J. Simmons, ${ }^{1}$ \\ and Linda J. Bendall ${ }^{3}$ \\ ${ }^{1}$ Stem Cell Biology Laboratory, Peter MacCallum Cancer Institute, Melbourne, Victoria, Australia \\ ${ }^{2}$ Fukuoka University School of Medicine, Fukuoka, Japan \\ ${ }^{3}$ Westmead Institute for Cancer Research, University of Sydney at Westmead Millennium Institute, Westmead, \\ New South Wales, Australia
}

\begin{abstract}
Hematopoietic progenitor cells (HPCs) normally reside in the bone marrow (BM) but can be mobilized into the peripheral blood (PB) after treatment with GCSF or chemotherapy. In previous studies, we showed that granulocyte precursors accumulate in the BM during mobilization induced by either GCSF or cyclophosphamide (CY), leading to the accumulation of active neutrophil proteases in this tissue. We now report that mobilization of HPCs by GCSF coincides in vivo with the cleavage of the $\mathrm{N}$-terminus of the chemokine receptor CXCR4 on HPCs resident in the BM and mobilized into the PB. This cleavage of CXCR4 on mobilized HPCs results in the loss of chemotaxis in response to the CXCR4 ligand, the chemokine stromal cell-derived factor-1 (SDF-1/CXCL12). Furthermore, the concentration of SDF-1 decreased in vivo in the BM of mobilized mice, and this decrease coincided with the accumulation of serine proteases able to directly cleave and inactivate SDF-1. Since both SDF-1 and its receptor, CXCR4, are essential for the homing and retention of HPCs in the BM, the proteolytic degradation of SDF-1, together with that of CXCR4, could represent a critical step leading to the mobilization of HPCs into the PB in response to GCSF or CY.
\end{abstract}

J. Clin. Invest. 110:187-196 (2003). doi:10.1172/JCI200315994.

\section{Introduction}

Hematopoietic stem and progenitor cells (HPCs) ensure the continuous renewal of mature blood cells. This rare population of cells has the unique property to engraft the bone marrow (BM) after lethal irradiation or chemotherapy and to fully reconstitute both the hematopoietic and immune systems (1). Until the early 1990s, hematopoietic rescue of patients receiving myeloablative chemotherapies was performed almost entirely with aspirates of BM as a source of transplantable HPCs. Currently, however, the great majority of

Received for publication May 24, 2002, and accepted in revised form December 4, 2002.

Address correspondence to: Jean-Pierre Lévesque, Peter MacCallum Cancer Institute, Stem Cell Biology Laboratory, Locked Bag 1, A'Beckett Street, Melbourne, VIC 8006, Australia. Phone: 61-3-9656-3717; Fax: 61-3-9656-3738;

E-mail: jp.levesque@pmci.unimelb.edu.au.

Conflict of interest: The authors have declared that no conflict of interest exists.

Nonstandard abbreviations used: hematopoietic progenitor cell (HPC); bone marrow (BM); peripheral blood (PB);

cyclophosphamide (CY); chemokine stromal cell-derived factor-1 (SDF-1/CXCL12); PB hematopoietic progenitor cell (PBPC); very late antigen-4 (VLA-4); phycoerythrin (PE); neutrophil elastase (NE); cathepsin G (CG); mean fluorescence intensity (MFI); PBS containing 0.05\% Tween-20 (PBST); batimastat (BB-94); $\mathrm{N}$-methoxysuccinyl-alanine-alanine-proline-valine-chloroform methylketone (MetOSuc-Ala-Ala-Pro-Val-CMK); N-methoxysuccinyl-alanine-alanine-phenylalanine- $\mathrm{PO}(\mathrm{O} \text {-phenyl })_{2}$

[MetOSuc-Ala-Ala-Phe-PO(Phe) $)_{2}$; paranitroanilide (pNA). transplants are performed using peripheral blood (PB) as a source of reconstituting cells. Although HPCs circulate at low to undetectable levels in steady-state PB, perturbations of the hematopoietic system, such as those resulting from myeloablative chemotherapy (2), or the administration of cytokines such as GCSF (3) lead to transient increases in the numbers of circulating HPCs, a phenomenon termed mobilization (4). The use of mobilized PB hematopoietic progenitor cells (PBPCs) is associated with more rapid engraftment, decreased morbidity, and reduced costs as compared with BM transplantation, all of which have contributed to the decline in the utilization of BM as a source of HPCs (4). Despite the now widespread use of mobilized PBPCs (around 30,000 transplants per year worldwide), the mechanisms that contribute to mobilization of primitive HPCs remain poorly understood.

The homing and retention of HPCs in the BM are controlled by adhesive interactions between HPCs and the BM stroma (5-8). The interaction between VCAM-1/CD106, which is expressed by BM stromal cells, and its counter-receptor integrin $\alpha 4 \beta 1$ or very late antigen-4 (VLA-4) expressed at the surface of HPCs is critical to the homing and retention of HPCs in the BM. Homozygous targeted deletion of the integrin $\alpha 4$ gene results in decreased hematopoiesis in the fetal liver of day 11 or 12 mouse embryos and decreased homing of myeloid and B lymphoid precursors in the spleen and BM in day 18 embryos $(6,9)$. In adult mice, pretreatment 
of wild-type HPCs with function-blocking anti-VLA-4 $\mathrm{mAbs}$ results in a profound reduction of donor HPCs homing to the BM of lethally irradiated recipients (10). Administration of function-blocking anti-VLA-4 and anti-VCAM-1 mAbs in rodents and nonhuman primates elicits HPC mobilization, suggesting an important role for these two molecules in mobilization (10-12). Recently, we have demonstrated that VCAM-1 expression is profoundly decreased in the BM of mice mobilized with GCSF or the chemotherapeutic cytotoxic drug cyclophosphamide $(\mathrm{CY})$ and that the decreases in VCAM- 1 expression and HPC mobilization are synchronized with the accumulation within the BM of neutrophil proteases that directly cleave VCAM-1 $(13,14)$.

A second pathway critical to the homing and retention of HPCs within the BM is the CXCR4/CXCL12 chemotactic axis. In vitro, the chemokine stromal cell-derived factor-1 (SDF-1/CXCL12) is a potent chemoattractant for primitive $\mathrm{BM} \mathrm{CD} 34^{+} \mathrm{CD} 38^{-}$cells that include candidate hematopoietic stem cells and express the CXCL12 receptor CXCR4 (15-17). CXCL12 is produced by the BM stroma and bone tissue as two isoforms, $\alpha$ and $\beta$, which differ by a four-residue extension at the $\mathrm{C}$-terminus in the $\beta$ isoform, and it is thought to form a decreasing gradient from the extravascular compartment of the $\mathrm{BM}$ toward the lumen of vessels irrigating this tissue (18). CXCL12 plays a key role during ontogeny of the hematopoietic system in inducing the migration of primitive HPCs from the fetal liver to the BM during fetal development $(19,20)$. In addition, in the adult, CXCL12 has been shown to promote engraftment of transplanted HPCs in the BM and subsequent hematopoietic reconstitution (21). The chemotactic effects of CXCL12 are mediated by the $G$ protein-linked receptor CXCR4, which upon ligand binding activates integrinmediated firm adhesion and transmigration of HPCs through the BM endothelium $(15,16,22)$.

Several groups, including our own, have proposed that the release of primitive hematopoietic cells into the peripheral circulation is the result of perturbation of adhesive interactions with BM stromal cell elements, which under steady-state conditions restrict these cells to the BM. We therefore hypothesized that the administration of mobilizing agents such as GCSF or the chemotherapeutic agent CY leads to the inactivation of the CXCL12/CXCR4 chemotactic pathway in the $\mathrm{BM}$, thereby facilitating their egress into the circulation (mobilization).

\section{Methods}

Mobilization of human patients and donors. Patients and healthy donors were mobilized by subcutaneous injection of $3 \mu \mathrm{g} / \mathrm{kg}$ of recombinant human GCSF (Filgastrim, Amgen Inc., Thousand Oaks, California, USA) once daily for 4 consecutive days or $5 \mu \mathrm{g} / \mathrm{kg}$ twice daily for 5 consecutive days.

Analysis of human CXCR4 degradation by immunofluorescence. Low-density mononucleated cells from $\mathrm{BM}$ aspirates from normal donors and from the PB of
GCSF-mobilized patients were isolated by centrifugation on a Ficoll-Hypaque (Amersham Pharmacia, Buckinghamshire, United Kingdom). Following three washes, cells were incubated on ice in the presence of $9 \mu \mathrm{g} / \mathrm{ml}$ mouse anti-human CXCR4 6H8 (IgG1), a mAb specific for an epitope located between residues 22 and 25 of human CXCR4 $(23,24)$, and $10 \mu \mathrm{g} / \mathrm{ml}$ mouse antihuman CD34 mAb 43A1 (IgG3). After two washes, cells were incubated with 1:200 dilutions of a biotinylated goat anti-mouse IgG3 and a phycoerythrin-conjugated (PE-conjugated) goat anti-mouse IgG1 (Southern Biotechnology, Birmingham, Alabama, USA). After two washes, cells were incubated with a 1:200 dilution of FITC-conjugated streptavidin (BD Pharmingen, San Jose, California, USA), washed, and analyzed by two-color flow cytometry. Alternatively, cells were labeled with 12G5, a mouse anti-human CXCR4 specific for the second extracellular domain $(23,24)$. For this purpose, cells were incubated with PE-conjugated 12G5 (R\&D Systems, Minneapolis, Minnesota, USA) and FITC-conjugated mouse anti-human CD34 antibody HPCA-2 (BD Pharmingen), washed, and analyzed on a FACScan flow cytometer (Becton-Dickinson, Mountain View, California, USA).

Proteolytic cleavage of CXCR 4 by purified neutrophil proteases was performed using the acute $\mathrm{B}$ lymphoid leukemia cell line Nalm- 6 or normal BM CD $34^{+}$cells. Nalm-6 cells in the exponential phase of culture or freshly isolated $\mathrm{BM} \mathrm{CD} 34^{+}$cells were washed three times in DMEM containing 0.2\% BSA and resuspended at $5 \times 10^{6}$ cells per milliliter. One hundred-microliter aliquots were mixed with increasing concentrations of neutrophil elastase (NE) and cathepsin G (CG) purified from human sputum (Elastin Products, Owensville, Missouri, USA) and incubated for 2 hours at $37^{\circ} \mathrm{C}$ with rotational shaking $(240 \mathrm{rpm})$. After digestion, all subsequent steps were performed on ice with ice-cold buffers. Cells were washed once with PBS containing 5\% FCS and incubated for 20 minutes with DMEM containing $10 \% \mathrm{FCS}$ and $10 \mu \mathrm{g} / \mathrm{ml}$ purified human immunoglobulins to block $F c$ receptors. Cells were then incubated for 40 minutes in the presence of $9 \mu \mathrm{g} / \mathrm{ml} \mathrm{mAb} 6 \mathrm{H} 8$. After two washes, cells were incubated with FITC-conjugated sheep F(ab)'2 fragment anti-mouse IgG (Silenus Labs, Boronia, Australia). Alternatively, after protease treatments, cells were labeled with PE-conjugated 12G5. After two washes, cells were resuspended in the presence of $20 \mu \mathrm{g} / \mathrm{ml}$ 7-amino actinomycin D. Results were expressed as a percentage of $6 \mathrm{H} 8$ or $12 \mathrm{G} 5$ binding on untreated cells according to the following formula: $100 \times\left(\mathrm{MFI}_{+ \text {protease }}\right.$ - $\left.\mathrm{MFI}_{\mathrm{IgG1}}\right) /\left(\mathrm{MFI}_{\text {no protease }}-\mathrm{MFI}_{\mathrm{IgG1}}\right)$, in which $\mathrm{MFI}_{\text {tprotease }}$ is the mean fluorescence intensity (MFI) of proteasetreated Nalm-6 labeled with $6 \mathrm{H} 8 / 12 \mathrm{G} 5, \mathrm{MFI}_{\text {no protease }}$ is the MFI of cells labeled with $6 \mathrm{H} 8 / 12 \mathrm{G} 5$ without protease treatment, and $\mathrm{MFI}_{\mathrm{IgG} 1}$ is the MFI of cells labeled with a nonimmune IgG1.

Immunobistochemistry on buman BM sections. Trephines were taken from the iliac crest of healthy donors before and on day 4 of GCSF administration and were formalin 
fixed, decalcified, and paraffin embedded as previously described (25). After dewaxing in xylene and ethanol, slides were rehydrated with ethanol containing increasing proportions of water and blocked for 2 hours at room temperature with PBS containing 0.05\% Tween-20 (PBST), $5 \%$ goat serum, and $10 \mu \mathrm{g} / \mathrm{ml}$ purified goat $\mathrm{IgG}$ (blocking buffer). Slides were then incubated overnight at $4^{\circ} \mathrm{C}$ with $5 \mu \mathrm{g} / \mathrm{ml} 6 \mathrm{H} 8$ or nonimmune mouse IgG1 in blocking buffer. After four washes with PBST, slides were incubated for 2 hours with a 1:200 dilution of human-adsorbed biotinylated goat anti-mouse $\operatorname{IgG}$ (Caltag Laboratories, Burlingame, California, USA) in blocking buffer. After four washes in PBST, slides were incubated for 1 hour in a 1:400 dilution of alkaline phosphatase-conjugated streptavidin (Amersham Pharmacia). Slides were then stained with fast red in the presence of levamisole and naphtol phosphate as previously described (13) and mounted with Aquamount (BDH Chemicals, Kilsyth, Australia).

Mobilization of mice. Eight- to 11-week-old female $\mathrm{balb} / \mathrm{C}$ mice were mobilized according to three distinct protocols. In the first protocol, GCSF alone, mice were injected subcutaneously twice daily with $250 \mu \mathrm{g} / \mathrm{kg}$ recombinant human GCSF (Filgrastim, Amgen Inc.) for 6 consecutive days. In the second protocol, CY alone, mice received a single intraperitoneal injection of $200 \mathrm{mg} / \mathrm{kg} \mathrm{CY}$ (Cycloblastin, Pharmacia and Upjohn, Rydalmere, Australia). In the third protocol, CY plus GCSF, mice received a single intraperitoneal injection of CY on day 0 (as in the second protocol) and were injected subcutaneously twice daily with 250 $\mu \mathrm{g} / \mathrm{kg}$ GCSF for the following 5 days. Some mice were then left to rest after day 6 to be sacrificed on day 10 . Control mice were either not injected or were injected with an equivalent volume of saline according to the same schedule. At indicated times, mice were sacrificed by cervical dislocation, and $\mathrm{PB}$, spleens, and femurs were collected as previously described (13). Clonogenic assays to measure the number of CFCs mobilized into the PB were performed as previously described (13).

Extraction of BM extracellular fluids and CXCL12 quantification. BM extracellular fluids were extracted from femurs into ice-cold PBS as previously described (13). Each BM extracellular fluid was diluted with an equal volume of buffer and further analyzed in duplicates with the commercial human CXCL12 ELISA kit from R\&D Systems. Calibration of the assay was performed with serial dilutions of recombinant human CXCL12 $\alpha$ provided in the kit. The relation between optical density and CXCL12 $\alpha$ concentration was linear from 34 to $5,000 \mathrm{pg} / \mathrm{ml}(P=0.9997)$.

Digestion of synthetic human CXCL12 $\alpha$. Medium conditioned by either human BM CD34- cells or PB neutrophils was prepared exactly as previously described (13). Aliquots of synthetic human CXCL12 $\alpha$ (kindly provided by Ian Clark-Lewis, University of British Columbia, Vancouver, Canada) at $20 \mu \mathrm{g} / \mathrm{ml}$ in Tris-buffered saline ( $\mathrm{pH}$ 7.4) containing $5 \mathrm{mM} \mathrm{CaCl}_{2}$ were incubated overnight at $37^{\circ} \mathrm{C}$ with an equal volume of $\mathrm{BM}$ extracellular fluids or conditioned medium or in the presence of $2 \mu \mathrm{g} / \mathrm{ml} \mathrm{NE}$, $10 \mu \mathrm{g} / \mathrm{ml} \mathrm{CG}$, or $2 \mu \mathrm{g} / \mathrm{ml}$ proteinase-3 purified from human sputum (Elastin Products). In some experiments, BM extracellular fluids were preincubated for 15 minutes at $37^{\circ} \mathrm{C}$ with either $0.5 \mathrm{mg} / \mathrm{ml}$ human $\alpha 1$-antitrypsin (Sigma-Aldrich, St Louis, Missouri, USA), 1 mM PMSF, $10 \mu \mathrm{M}$ batimastat (BB-94) (British Biotech, Oxford, United Kingdom), $10 \mu \mathrm{M}$ N-methoxysuccinyl-alanine-alanine-proline-valine-chloroform methylketone (MetOSucAla-Ala-Pro-Val-CMK; Calbiochem-Novabiochem, San Diego, California, USA), or $10 \mu \mathrm{M} \mathrm{N}$-methoxysuccinylalanine-alanine-phenylalanine- $\mathrm{PO}(\mathrm{O}-\mathrm{phenyl})_{2}$ [MetOSuc-Ala-Ala-Phe-PO(Phe) 2 ; catalog number DAP-22, Enzyme Systems Products, Livermore, California, USA) before incubation with human CXCL12 $\alpha$.

Transmigration assays. The remaining chemotactic activity after digestion of synthetic human CXCL12 $\alpha$ was measured using $\mathrm{CD} 34^{+}$cells freshly isolated from normal human BM (26) or Nalm- 6 cells as previously described (27). Cells $\left(5 \times 10^{4}\right)$ were plated in $8-\mu \mathrm{m}$ pore transwell inserts (Corning Costar, Cambridge, Massachusetts, USA). Exogenous CXCL12 $\alpha$ preincubated with the indicated mouse BM extracellular fluids was added in the lower chamber at a concentration corresponding to 200 $\mathrm{ng} / \mathrm{ml}$ intact CXCL12 $\alpha$ before digestion. After 4 hours at $37^{\circ} \mathrm{C}$, the percentage of cells that had transmigrated to the lower chamber was determined by flow cytometry.

Analysis of CXCL12 degradation by Western blot. Degradation of CXCL12 was analyzed by Western blot after boiling $5-\mu \mathrm{l}$ aliquots of digested synthetic human CXCL12 $\alpha$ for 3 minutes in an equal volume of electrophoresis sample buffer containing $10 \mathrm{mM}$ DTT. Samples were electrophoresed on a $20 \%$ polyacrylamide Tris-Trycine-SDS gel. Following transfer to nitrocellulose, membranes were blocked overnight in PBS containing $0.05 \%$ Tween- 20 and 3\% BSA. Membranes were then incubated sequentially in the presence of 100 $\mathrm{ng} / \mathrm{ml}$ goat anti-human CXCL12 $\alpha$ serum (R\&D Systems), a 1:10,000 dilution of biotinylated donkey $\mathrm{F}(\mathrm{ab})$ '2 fragment anti-goat IgG (Jackson ImmunoResearch Laboratories Inc., West Grove, Pennsylvania, USA), and a 1:4,000 dilution of streptavidin-biotinylated horseradish peroxidase complex (Amersham Pharmacia) in blocking buffer and revealed by enhanced chemiluminescence.

Statistical analyses. Levels of significance were measured using Students' two-tailed non-paired $t$ test.

\section{Results}

Mobilized human $P B C D 34^{+}$cells express a truncated form of CXCR4. Previous reports have shown that neutrophil proteases NE and CG accumulate in the BM of humans and mice mobilized with $\operatorname{GCSF}(13,14)$ and that human CXCR4 is cleaved in vitro by purified NE (24). This prompted us to determine whether CXCR4 expressed by HPCs could be cleaved in vivo during the process of mobilization in response to GCSF administration. In a first set of experiments, we analyzed the binding of the $\mathrm{mAb} 6 \mathrm{H} 8$, which is specific for residues $22-25$ of human 
a

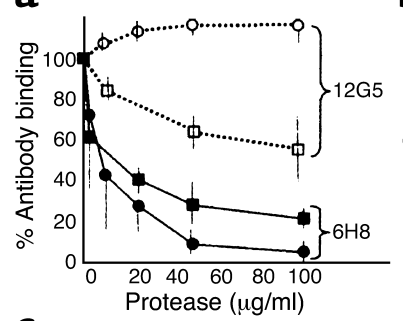

C

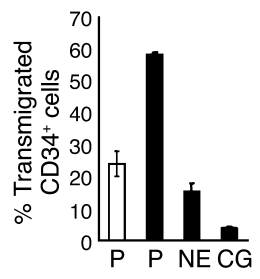

d

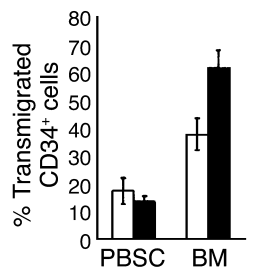

b

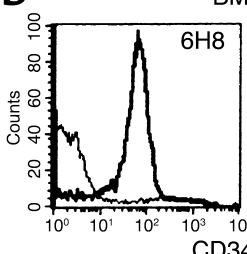

$\mathrm{BM}$ CD34+
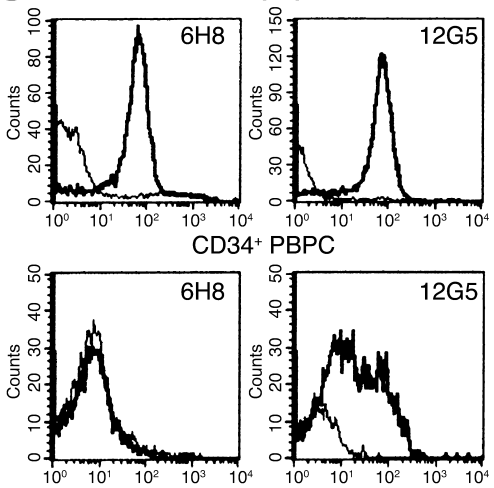

CD34+ PBPC cultured
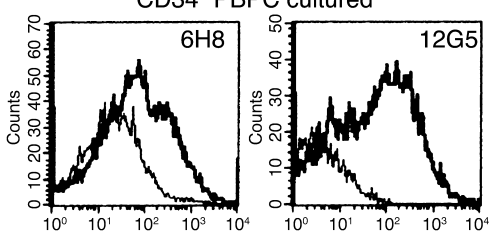

Figure 1

Mobilized CD34 ${ }^{+}$PBPCs express a CXCR4 molecule truncated in the first extracellular domain. (a) Nalm- 6 cells were incubated for 2 hours at $37^{\circ} \mathrm{C}$ in the presence of indicated concentrations of purified NE (circles) or CG (squares) and were stained with the mAbs $6 \mathrm{H} 8$ or $12 \mathrm{G} 5$. After analysis by flow cytometry, results are expressed as a percentage of $6 \mathrm{H} 8 / 12 \mathrm{G} 5$ binding of nontreated cells. Data represent means \pm SD of three independent experiments. (b) Binding of $6 \mathrm{H} 8$ and $12 \mathrm{G} 5 \mathrm{mAbs}$ to steady-state $\mathrm{CD}_{4}{ }^{+} \mathrm{BM}$ cells, GCSF-mobilized CD34 ${ }^{+}$PBPCs, and GCSF-mobilized CD34+ PBPCs after overnight culture. The flow cytometry analysis was gated on $\mathrm{CD}_{3} 4^{+}$cells. Representative data from two experiments are shown. (c) $\mathrm{CD} 34^{+}$cells isolated from normal steady-state BM were treated with $100 \mu \mathrm{g} / \mathrm{ml}$ of NE or CG. Control cells were pretreated in an identical manner with PBS in the absence of protease. The chemotactic response of cells was assessed in the presence (black bars) or absence (white bars) of $200 \mathrm{ng} / \mathrm{ml}$ of CXCL12 in the lower chamber. Representative data from two experiments in triplicate are shown. P, PBS. (d) Freshly isolated GCSF-mobilized CD34+ PBPCs or CD34+ cells derived from steady-state BM were compared for their chemotactic response in the absence (white bars) and presence (black bars) of $200 \mathrm{ng} / \mathrm{ml} \mathrm{CXCL12.} \mathrm{Representative} \mathrm{data} \mathrm{from}$ two experiments are shown.
CXCR4 $(23,24)$ - a site located within the first N-terminal extracellular domain of CXCR4 - on acute B lymphoid leukemia cell line Nalm-6 after treatment with increasing concentrations of purified NE and CG. As shown in Figure 1a, both proteases induced a dosedependent reduction of $6 \mathrm{H} 8$ binding to Nalm- 6 cells, whereas the binding of $\mathrm{mAb} 12 \mathrm{G} 5$, which recognizes an epitope located within the second extracellular domain of CXCR4 $(23,24)$, was increased by $20 \%$ after treatment with NE and decreased by $40 \%$ after treatment with CG. These data confirm that both NE and CG can cleave between the $6 \mathrm{H} 8$ epitope and the first transmembrane domain of CXCR4, a cleavage known to inactivate the chemotactic properties of CXCR4 $(23,24)$.

In a second set of experiments, we followed the binding of $6 \mathrm{H} 8$ and $12 \mathrm{G} 5 \mathrm{mAbs}$ to $\mathrm{CD} 4^{+}$cells isolated from steady-state BM and GCSF-mobilized PB (Figure 1b). Although all CD34+ cells isolated from steady-state $\mathrm{BM}$ were stained brightly by either $6 \mathrm{H} 8$ or $12 \mathrm{G} 5 \mathrm{mAbs}$, $\mathrm{CD} 4^{+}$cells from GCSF-mobilized PB were characterized by a complete lack of $6 \mathrm{H} 8$ binding despite lower but still positive staining with $12 \mathrm{G} 5$. Overnight culture of purified $\mathrm{CD} 34^{+}$mobilized PBPCs resulted in reexpression of the $6 \mathrm{H} 8$ epitope (Figure $1 \mathrm{~b})$. These data do not rule out the possibility that the partial decrease of $12 \mathrm{G} 5$ binding may be due to reduced transcription of the CXCR4 mRNA. However, the fact the mAb $6 \mathrm{H} 8$ failed to bind to mobilized CD $34^{+}$PBPCs that were still positive for $12 \mathrm{G} 5$ demonstrates that, like Nalm- 6 cells treated in vitro with purified $\mathrm{NE}$ and $\mathrm{CG}, \mathrm{CD} 34^{+}$ PBPCs mobilized in vivo with GCSF express a truncated form of CXCR4 containing the second transmembrane domain but lacking at least the $25 \mathrm{~N}$-terminal residues in the first extracellular domain.
Loss of CXCR4 N-terminus in GCSF-mobilized BM. Immunohistochemical stains were performed with $\mathrm{mAb}$ $6 \mathrm{H} 8$ on human $\mathrm{BM}$ sections taken before and on day 4 of GCSF administration (Figure 2). Before GCSF administration, $6 \mathrm{H} 8$ binding was particularly strong in the endosteal region in the vicinity of trabecular bone, where the most primitive HPCs reside. On day 4 of GCSF administration, staining for $6 \mathrm{H} 8$ was greatly reduced, showing that the truncation of CXCR4 is not only seen on $\mathrm{CD}_{3} 4^{+}$cells once they are mobilized into the PB but also occurs in the BM when GCSF is administered.
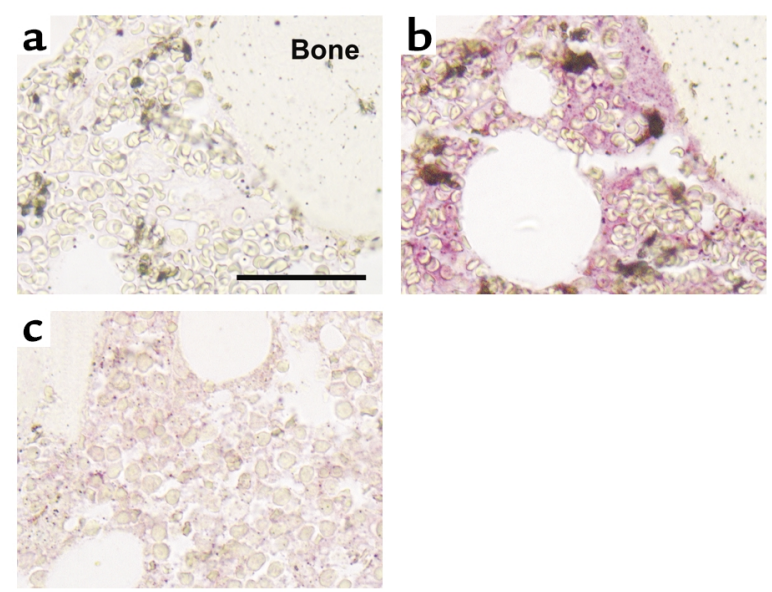

\section{Figure 2}

$\mathrm{BM}$ loses reactivity for anti-human CXCR4 mAb $6 \mathrm{H} 8$ during mobilization with GCSF. BM sections taken before mobilization were stained with a nonimmune $\lg \mathrm{G} 1$ (a) and $\mathrm{mAb} 6 \mathrm{H} 8$ (b and $\mathbf{c}$ ). Positive staining appears in purple (magnification, $\times 400$ ). $6 \mathrm{H} 8$ staining on BM on day 4 of GCSF administration was very $\operatorname{dim}(\mathbf{c})$. The scale bar represents $50 \mu \mathrm{m}$. 
GCSF-mobilized CD34+ PBPCs do not migrate toward CXCL12. The cleavage of the $\mathrm{N}$-terminal CXCR 4 by NE has previously been reported to reduce binding of CXCL12 to Jurkat $\mathrm{T}$ cells to undetectable levels and to abolish the chemotactic response to CXCL12 gradient in vitro (24). We therefore examined the effect of exposure to NE or CG on the chemotactic response of steady-state $\mathrm{BM} \mathrm{CD} 34^{+}$cells that expressed $6 \mathrm{H} 8$ epitope before in vitro exposure to proteases. Both NE and CG treatments resulted in the complete inhibition of CXCL12-driven chemotaxis of CD34 $4^{+}$progenitors (Figure 1c), in good accord with our finding that treatment with either protease resulted in the complete loss of $6 \mathrm{H} 8$ epitope (Figure 1a). Since GCSF-mobilized CD34 ${ }^{+}$ PBPCs lack the $6 \mathrm{H} 8$ epitope (Figure $1 \mathrm{~b}$ ), we then investigated the chemotactic response of freshly isolated CD34 $4^{+}$PBPCs to CXCL12. In contrast to the robust chemotactic response observed with $\mathrm{CD} 34^{+}$cells isolated from steady-state BM, GCSF-mobilized CD34 ${ }^{+}$ PBPCs failed to respond to CXCL12 (Figure 1d).

Decrease of CXCL12 concentration in mouse BM extracellular fluids during mobilization. We next investigated whether the CXCR4 ligand CXCL12 may be also be degraded in mobilized BM using the murine model in which we demonstrated accumulation of active NE and CG during mobilization of HPCs induced by GCSF or CY $(13,14)$. For this purpose, balb/c mice were mobilized after injection of either GCSF alone, CY alone, or CY in combination with GCSF. At various time points, BM extracellular fluids were extracted, and the concentration of endogenous murine CXCL12 was determined by ELISA. Concentrations of CXCL12 in these $\mathrm{BM}$ extracellular extracts were significantly decreased on day 6 after administration of CY alone or CY plus GCSF (Figure 3a). This corresponded precisely to the time at which HPCs were mobilized into the PB (Figure $3 b)$. Similarly, in animals receiving GCSF alone, CXCL12 concentration in the BM was significantly decreased between days 2 and 6 of cytokine administration, again corresponding to the time of maximal HPC numbers in the PB. On day 10, CXCL12 concentrations in the $\mathrm{BM}$ returned to levels seen before the initiation of mobilization, and there was a concordant decrease in the number of circulating HPCs. It is interesting to note that on day 3 after injection of $\mathrm{CY}$ alone or CY plus GCSF, when mice are neutropenic and HPCs are not mobilized into the PB (14), CXCL12 concentration in the BM was significantly increased as compared with steady-state BM. Thus, CXCL12 levels in the BM are inversely related to the numbers of PBPCs in mice receiving three different mobilization regimens involving either a cytokine alone (GCSF), chemotherapy alone, or the combination of both.

$B M$ fluids from mobilized mice contain proteases inactivating CXCL12. We have previously reported that active neutrophil proteases accumulate in the BM extracellular fluid during mobilization and that these proteases cleave VCAM-1, which is essential to the retention of HPCs in the $\mathrm{BM}(13,14)$. On the basis of this observation, we hypothesized that the decrease of endogenous CXCL12 concentration in the BM of mobilized mice could be due to proteolytic degradation. Since the BM from a 8- to 11-week-old mouse femur represents a total volume of $10 \mu \mathrm{l}$ (approximately $90-95 \%$ cells and $5-10 \%$ fluid) and is flushed into $1 \mathrm{ml}$ of PBS, our BM extracellular fluids were consequently diluted between 500 and 1,000 times during the extraction process. Since a concentration of at least $10 \mathrm{ng} / \mathrm{ml}$ CXCL12 is required to promote chemotaxis in CD34 ${ }^{+}$cells (16), 400 pg of endogenous mouse CXCL12 contained within the BM of one femur and diluted into $1 \mathrm{ml}$ of PBS represented a concentration too low to induce chemotaxis in vitro. Therefore, to assess the possibility that proteases cleaving and inactivating CXCL12 were released in mobilized BM, synthetic human CXCL12 $\alpha$ (which is identical to mouse CXCL12 $\alpha$ except for a Val to Ile substitution in position 18) was incubated at $37^{\circ} \mathrm{C}$ with the $\mathrm{BM}$ extracellular fluids extracted from mice at different time points of mobilization. The residual bioactivity of the exogenous synthetic human CXCL12 $\alpha$ was subsequently evaluated in transmigration assays on $\mathrm{CD} 34^{+}$cells isolated from steady-state human BM and on Nalm- 6 cells. BM extracellular fluids without exogenous CXCL12 $\alpha$ were used to control basal transmigration. Synthetic CXCL12 $\alpha$ preincubated in the presence of PBS was used as a positive control.

As anticipated, BM extracellular fluids in the absence of exogenous synthetic CXCL12 $\alpha$ were unable to promote chemotaxis due to the 500- to 1,000-fold dilution of endogenous murine CXCL12 (Figure 4a, white bars), whereas exogenous synthetic human CXCL12 $\alpha$ preincubated with either PBS or BM extracellular
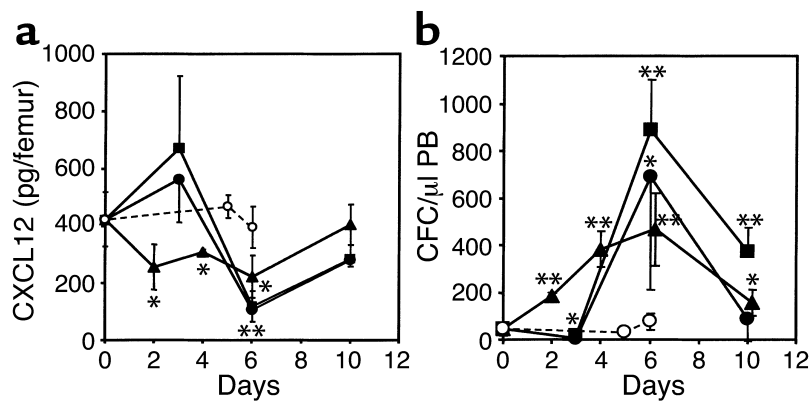

\section{Figure 3}

CXCL12 concentration in the BM decreases when HPCs are mobilized in the PB. (a) BM extracellular fluids were extracted at the indicated time points from mice injected with either saline (open circles), $\mathrm{CY}$ alone (filled circles), GCSF alone (filled triangles), or CY in combination with GCSF (filled squares). CXCL12 concentrations were quantified by ELISA. (b) PB from mice injected with either $C Y$ alone (filled circles), GCSF alone (filled triangles), CY in combination with GCSF (filled squares), or saline (open circles) was taken at the indicated time points and plated in triplicate in clonogenic assays. The numbers of CFCs were determined after 14 days of incubation at $37^{\circ} \mathrm{C}$. Data are means \pm SD of three to six mice per group, with each sample analyzed in triplicate. Statistically significant differences with noninjected animals are indicated $\left({ }^{*} P<0.05,{ }^{*} P<0.01\right.$, as determined by Student's $t$ test). 


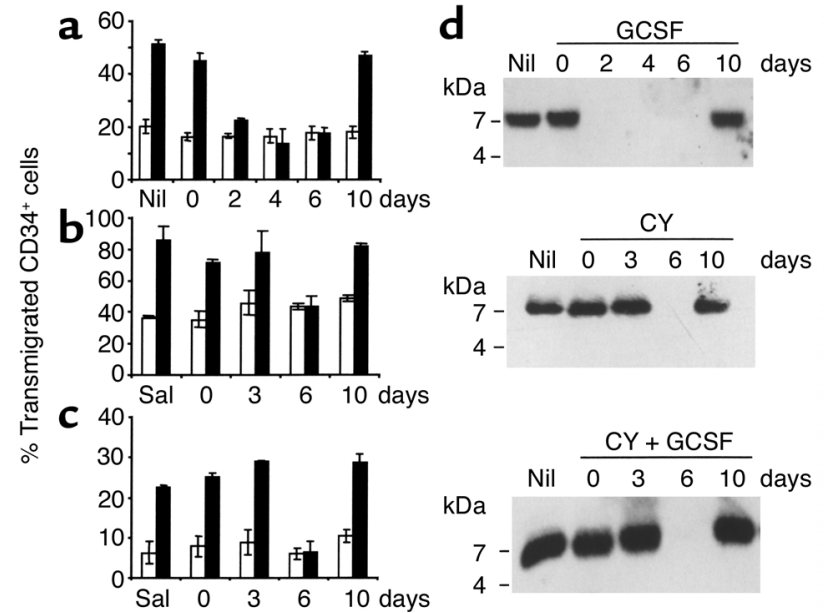

Figure 4

BM extracellular fluids from mobilized mice contain proteases cleaving exogenous human synthetic CXCL12 $\alpha$. (a-c) Aliquots of exogenous synthetic human $\mathrm{CXCL} 12 \alpha$ were incubated overnight at $37^{\circ} \mathrm{C}$ in the presence of an equal volume of BM extracellular fluids taken from mice mobilized with either GCSF alone (a), CY alone (b), or CY in combination with GCSF (c). The remaining chemotactic activity of exogenous CXCL12 $\alpha$ was measured by performing transmigration assays with $\mathrm{CD} 34^{+}$cells freshly isolated from normal human BM. Nil indicates that PBS was added instead of BM extracellular extracts. In b and $\mathbf{c}$, Sal represents the BM extracellular fluid from mice injected with saline for 6 days. Black bars show transmigration in the presence of digested CXCL12 $\alpha$, whereas white bars show controls in which exogenous CXCL12 $\alpha$ was omitted. Data represent means \pm SD of duplicates. Representative data from three independent experiments are shown. (d) The same samples of synthetic human CXCL12 $\alpha$ incubated with BM extracellular fluids (as in a) were electrophoresed on a $20 \%$ polyacrylamide Tris-Trycine-SDS gel and analyzed by Western blotting with a goat anti-human CXCL12 $\alpha$ antibody. A representative experiment from three performed is shown.

fluids from uninjected mice induced chemotaxis of human steady-state CD34+ BM cells (Figure 4a, black bars). However, preincubation of synthetic CXCL12 $\alpha$ with BM extracts isolated between days 2 and 6 of GCSF mobilization completely inactivated the chemotactic activity of exogenous CXCL $12 \alpha$ on $\mathrm{CD} 34^{+}$cells isolated from steady-state BM. Similarly, incubation of synthetic CXCL12 $\alpha$ with BM extracts isolated on day 6 after injection of either CY or CY plus GCSF, when HPC mobilization peaked, completely inactivated the chemotactic activity of exogenous CXCL12 $\alpha$

\footnotetext{
Figure 5

Cleavage and inactivation of CXCL12 in mobilized BM is due to serine proteases. Aliquots of synthetic human CXCL12 $\alpha$ were incubated overnight at $37^{\circ} \mathrm{C}$ in the presence of PBS (lane 1), BM extracellular fluids isolated on day 4 of GCSF mobilization (lanes 2-5) after preincubation in the absence of protease inhibitor (lane 2) or in the presence of human $\alpha 1$-antitrypsin (lane 3), PMSF (lane 4), or BB-94 (lane $5)$. In the top panel, samples (black bars) together with controls without exogenous CXCL12 $\alpha$ were analyzed for chemotactic activity on Nalm- 6 cells as described in Figure 4a. A representative experiment from two performed is shown. In the bottom panel, the same samples were analyzed by Western blot with a goat anti-human CXCL12 antibody. A representative experiment from two performed is shown.
}

(Figure 4a). Results on Nalm-6 cells showed the same result with a total inactivation of CXCL12 $\alpha$ chemotactic activity after incubation with BM extracts taken between days 2 and 6 of GCSF-induced mobilization or on day 6 of CY-induced or CY plus GCSF-induced mobilization (data not shown).

When these digests were analyzed by immunoblotting, exogenous CXCL12 $\alpha$ was no longer detectable after incubation with BM extracellular fluids from mice during HPC mobilization - that is, between days 2 and 6 of GCSF-induced mobilization (Figure 4a) and on day 6 of mobilization with $\mathrm{CY}$ alone (Figure $4 \mathrm{~b}$ ) or in combination with GCSF (Figures 4c). On day 10, when mobilization had ceased (Figure 3b), none of the $\mathrm{BM}$ extracellular fluids inactivated or degraded the exogenous CXCL12 $\alpha$ (Figure 4).

To assess whether this inactivation of exogenous CXCL12 $\alpha$ was due to proteases present in the BM extracellular fluids, BM extracellular fluids extracted on day 4 of mobilization with GCSF were preincubated with human $\alpha 1$-antitrypsin (tissue inhibitor of serine proteases), PMSF (a serine protease inhibitor) or BB-94 (a broad specificity inhibitor of matrix metalloproteinases) $(28,29)$ before addition to synthetic CXCL12 $\alpha$. As shown in Figure 5, pretreatment of GCSF-mobilized BM extracellular fluids with human $\alpha 1$-antitrypsin or PMSF completely abolished inactivation and degradation of exogenous synthetic CXCL12 $\alpha$ in both transmigration assays and Western blots, whereas pretreatment with BB-94 had no effect. This result demonstrates that proteases released in mobilized BM and responsible for CXCL12 $\alpha$ degradation and inactivation are serine proteases.

Neutrophils release in the BM proteases that inactivate CXCL12. To determine which cell population within the $\mathrm{BM}$ was responsible for the release of these proteases, synthetic human CXCL12 $\alpha$ was incubated with media conditioned either by BM CD34mononucleated cells or PB neutrophils and further analyzed in transmigration assays and by Western blotting (Figure 6). Incubation with either PB neutrophil-conditioned or BM CD34- cell-conditioned media abolished the chemotactic activity of exogenous synthetic CXCL12 $\alpha$. This result was confirmed by immunoblotting, which showed that these two conditioned media completely degraded CXCL12 $\alpha$. 


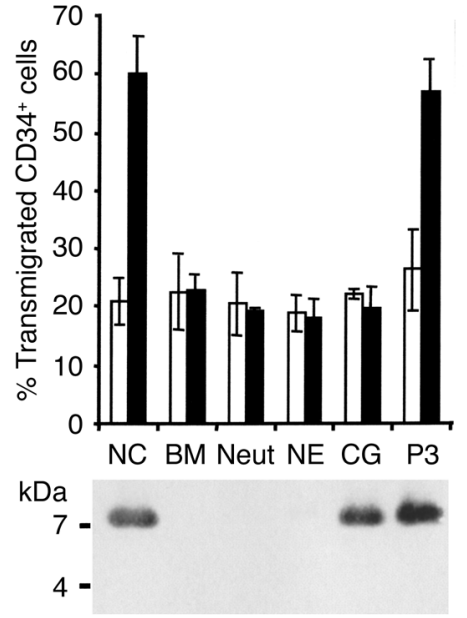

In contrast, neither BM stromal cell-conditioned nor bone cell-conditioned media degraded CXCL12 $\alpha$ (data not shown). This result demonstrates that neutrophils are a major source of proteases with the ability to degrade and inactivate CXCL12 $\alpha$.

Active NE and CG cleave and inactivate CXCL12. Since $\mathrm{NE}, \mathrm{CG}$, and proteinase- 3 are the three major serine proteases produced and released by neutrophils (30), we tested whether these enzymes were capable of inactivating the chemotactic activity of exogenous human CXCL12 $\alpha$ as assessed using the CD34+ cell transmigration assay. These experiments demonstrated that both NE and CG inactivated CXCL12 $\alpha$, whereas proteinase- 3 , a neutrophil serine protease closely related to $\mathrm{NE}$ (30), did not (Figure 6). In accord with this finding, CXCL12 $\alpha$ was no longer detected by Western blot analysis after digestion with NE, suggesting that it was digested into

\section{Figure 7}

Pretreatment of BM extracellular fluids from mobilized mice with a specific NE inhibitor together with a specific CG inhibitor prevents degradation and inactivation of CXCL12. Aliquots of synthetic human CXCL12 $\alpha$ were incubated overnight at $37^{\circ} \mathrm{C}$ in the presence of BM extracellular fluids on day 4 of GCSF-induced mobilization and day 6 of CY-induced mobilization that were pretreated with $1 \mathrm{mM}$ PMSF, $10 \mu \mathrm{M}$ specific NE inhibitor MetOSuc-Ala-Ala-Pro-Val-CMK, or $10 \mu \mathrm{M}$ specific CG inhibitor MetOSuc-Ala-Ala-Phe-PO(Phe $)_{2}$ alone or in combination. In the top panel, the remaining chemotactic activity of exogenous human CXCL12 $\alpha$ was measured on purified Nalm- 6 cells in transmigration assays as described in Figure 4a. A representative experiment from two performed in triplicate is shown. The bottom panel shows Western blot analysis of the same samples with a goat anti-human CXCL12 antibody. G4, day 4 of GCSF-induced mobilization; CY6, day 6 of CYinduced mobilization.

\section{Figure 6}

Neutrophil proteases NE and CG cleave and inactivate CXCL12 $\alpha$. Aliquots of synthetic human CXCL12 $\alpha$ were incubated overnight at $37^{\circ} \mathrm{C}$ in the presence of medium conditioned by either human BM CD34- cells, PB neutrophils, or nonconditioned medium. In parallel, CXCL12 $\alpha$ was also incubated with purified human NE, CG, or proteinase-3. In the top panel, the remaining chemotactic activity of exogenous human CXCL12 $\alpha$ was measured on purified BM CD34 ${ }^{+}$ cells in transmigration assays as described in Figure 4a. A representative experiment from three performed in triplicate is shown. The bottom panel shows Western blot analysis of the same samples with a goat anti-human CXCL12 antibody. BM, BM CD34- cells; Neut, PB neutrophils; NC, nonconditioned medium; P3, proteinase-3.

small fragments. In contrast, after digestion with CG, CXCL12 $\alpha$ exhibited a slightly faster electrophoretic mobility when overrun on a $20 \%$ SDS-PAGE (data not shown). These findings are consistent with the previous observation that CG cleaves CXCL12 $\alpha$ between the fifth and sixth residues from the $\mathrm{N}$-terminus, resulting in its complete inactivation (31).

Combination of NE- and CG-specific inhibitors prevents inactivation of CXCL12 by mobilized BM extracellular flu$i d s$. Proof that the proteases released in BM extracellular fluids during mobilization are NE and CG was provided by preincubating BM extracellular fluids on day 4 of GCSF-induced mobilization and day 6 of CY-induced mobilization with the specific NE inhibitor MetOSuc-Ala-Ala-Pro-Val-CMK and the specific CG inhibitor MetOSuc-Ala-Ala-Phe$\mathrm{PO}(\mathrm{Phe})_{2}$. A preliminary experiment using NE and

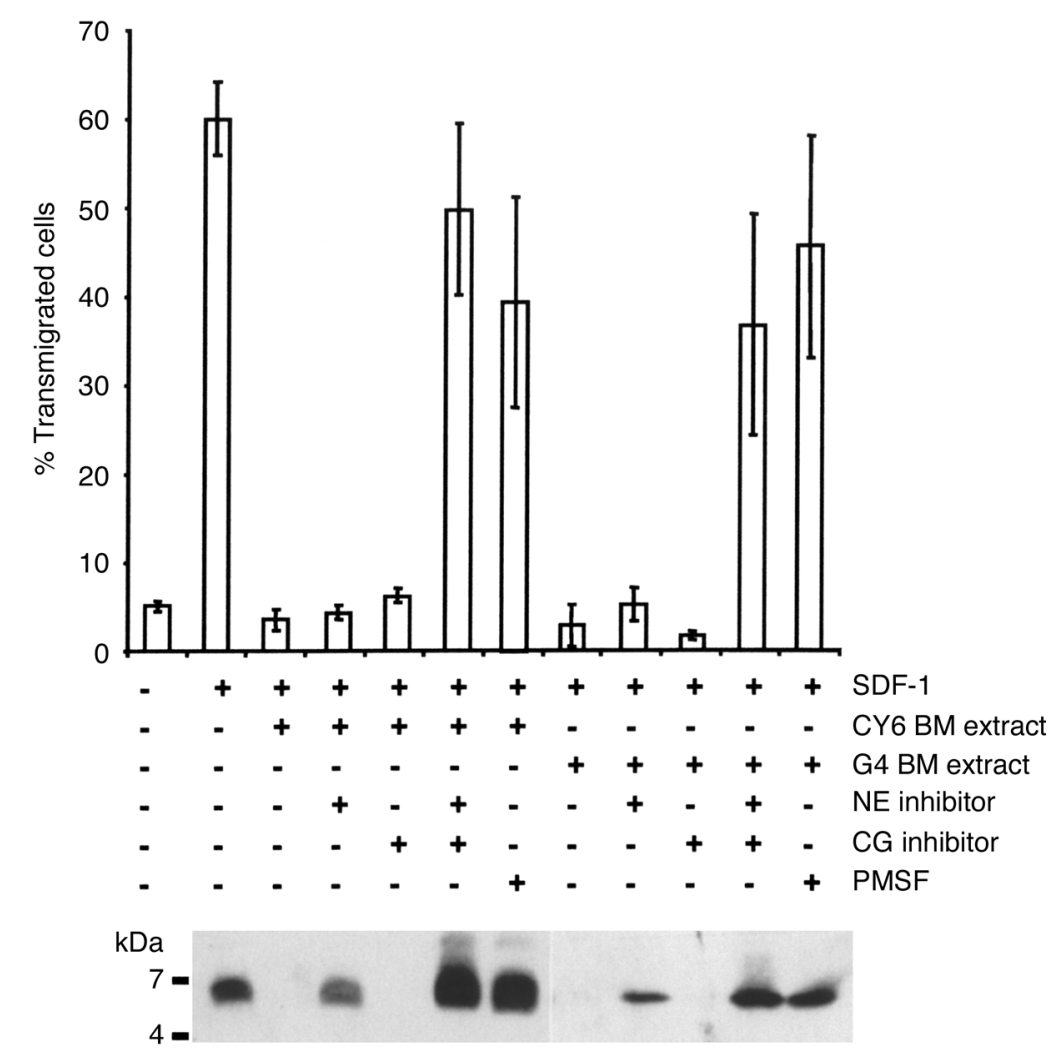


CG purified from human sputum and the chromogenic substrates MetOSuc-Ala-Ala-Pro-Val paranitroanilide (pNA) and Suc-Ala-Ala-Pro-Phe-pNA, which are specific for NE and CG, respectively (13, 14), demonstrated unambiguously that MetOSucAla-Ala-Pro-Val-CMK inhibits NE but not CG, whereas MetOSuc-Ala-Ala-Phe-PO(Phe $)_{2}$ inhibits CG but not NE (data not shown). Pretreatment of the BM extracellular fluids with MetOSuc-Ala-Ala-Pro-ValCMK alone significantly reduced the degradation of CXCL12 $\alpha$, as observed by Western blotting (Figure $7 \mathrm{~b}$ ), but had a marginal effect on the inactivation of the CXCL12 $\alpha$ chemotactic activity (Figure $7 a$ ), suggesting that a protease different from $\mathrm{NE}$ was present and, like CG, could inactivate CXCL12 activity with a minor shortening of the molecule. MetOSuc-AlaAla-Phe- $\mathrm{PO}(\mathrm{Phe})_{2}$ alone did not prevent inactivation of the chemotactic activity nor the degradation of CXCL12. However, preincubation of BM extracellular fluids with both MetOSuc-Ala-Ala-Pro-Val-CMK and MetOSuc-Ala-Ala-Phe-PO(Phe $)_{2}$ prevented the inactivation and proteolytic degradation of CXCL12 $\alpha$ by either CY-mobilized or GCSF-mobilized BM extracellular fluids.

\section{Discussion}

In previous studies, we have shown that mobilization by either GCSF or CY is accompanied by (1) a sharp increase in granulocytic precursors and mature granulocytes in the BM, (2) a release of large amounts of active neutrophil serine proteases such as NE and CG, and (3) a loss of VCAM- 1 expression in the extravascular compartment of the BM due to proteolytic cleavage by these neutrophil proteases $(13,14)$. The current study provides evidence that the chemokine receptor CXCR 4 expressed by human $\mathrm{CD} 34^{+} \mathrm{HPCs}$ is cleaved and truncated within the $\mathrm{BM}$ in vivo during mobilization induced by GCSF. Flow cytometry analyses demonstrated that $\mathrm{CD} 34^{+}$cells mobilized into the PB failed to bind the $\mathrm{mAb} 6 \mathrm{H} 8$, which recognizes an epitope located between residues 22 and 25 of the $\mathrm{N}$-terminal extracellular domain of human CXCR4, but still bound mAb 12G5, which binds to the second extracellular domain of human CXCR4, whereas $\mathrm{CD} 34^{+}$cells isolated from steady-state BM were brightly stained by both antibodies. In vitro experiments with the Nalm-6 cell line showed that exposure to the neutrophil proteases NE or CG, which both accumulate in large amounts in GCSF-mobilized BM $(13,14)$, resulted in a similar loss of $6 \mathrm{H} 8$ binding. Whereas NE treatment did not decrease 12G5 binding, CG treatment significantly reduced $12 \mathrm{G} 5$ binding to Nalm- 6 cells. This is consistent with the reduced but still significant binding of $12 \mathrm{G} 5$ to mobilized CD34+ PBPCs as compared with steady-state BM cells. In accord with previous findings showing that the presence of the $6 \mathrm{H} 8$ epitope is absolutely necessary to CXCR 4 chemotactic function (23), we show that mobilized CD $34^{+}$PBPCs migrated poorly in vitro in response to CXCL12 as compared with steady-state $\mathrm{BM} \mathrm{CD} 34^{+}$cells. Furthermore, in vitro treatment of steady-state $\mathrm{BM} \mathrm{CD} 34^{+}$cells with either NE or CG decreased both 6H8 expression and chemotactic response to CXCL12, as observed with GCSF-mobilized CD $34^{+}$PBPCs. These data indicate that the specific loss of $6 \mathrm{H} 8$ binding on mobilized CD $34^{+}$PBPCs is sufficient to cause the loss of chemotactic response to CXCL12.

Although a splicing variant with additional amino acids at the N-terminus of both human and mouse CXCR4 has been reported $(32,33)$, there is no evidence of a splicing variant lacking the $\mathrm{N}$-terminal residues 22-25 that form the 6H8 epitope of human CXCR4 (24). Therefore, the loss of the $6 \mathrm{H} 8$ epitope in mobilized CD34+ PBPCs is likely to be due to a proteasedependent truncation of the CXCR4 receptor molecule by proteolytic cleavage between the epitope recognized by $6 \mathrm{H} 8$ and the first transmembrane domain by NE and CG, two proteases that are both released in large amounts in the BM during mobilization by either $\operatorname{GCSF}$ or CY $(13,14)$.

The view that the loss of $6 \mathrm{H} 8$ expression and CXCR4 function is mediated in vivo by proteases released in the BM during mobilization is supported by the fact that (1) immunohistochemical stains of human $\mathrm{BM}$ confirmed that although $6 \mathrm{H} 8$ reacts strongly in nonmobilized BM cells, $6 \mathrm{H} 8$ reactivity is notably decreased in GCSF-mobilized BM (Figure 2), and that (2) an overnight incubation of GCSF-mobilized $\mathrm{CD} 34^{+} \mathrm{PBPCs}$ at $37^{\circ} \mathrm{C}$ in the absence of proteases allows re-expression of intact CXCR4 bearing the $6 \mathrm{H} 8$ epitope (Figure $1 \mathrm{~b}$ ). Of note, Petit et al. have reported an increase in CXCR4 expression on BM cells after GCSF administration (34). However, the antibody used in this study does not recognize the $\mathrm{N}$-terminal region of CXCR4 and so would not detect the truncation and loss of this functionally critical region of the molecule.

In a second series of experiments performed in a mouse model, we demonstrate that the concentration of CXCR4 ligand, the chemokine CXCL12, significantly decreases in BM extracellular fluids from mice mobilized by either GCSF or CY. Using synthetic exogenous human CXCL12 $\alpha$ as a target to measure whether BM extracellular fluids acquire the potential to degrade and inactivate endogenous CXCL12, we demonstrate that precisely when HPCs are mobilized into the $\mathrm{PB}$, neutrophil serine proteases able to directly degrade and inactivate the chemotactic activity of CXCL12 are released in BM extracellular fluids (between days 2 and 6 of GCSF-induced mobilization and on day 6 of CY-induced or CY plus GCSF-induced mobilization). This timing coincides precisely with the release of NE and CG in mobilized BM (14), two neutrophil proteases with the capability to directly degrade and inactivate CXCL12 chemotactic activity. Furthermore, the fact that the combination of the specific NE inhibitor MetOSuc-Ala-Ala-Pro-Val-CMK 
with the specific CG inhibitor MetOSuc-Ala-Ala-Phe$\mathrm{PO}(\mathrm{Phe})_{2}$ was able to block CXCL12 degradation and inactivation by $\mathrm{BM}$ extracellular fluids from CY- and GCSF-mobilized mice clearly demonstrates that these two proteases, which are released in the BM extracellular fluid of mobilized mice, are responsible for the degradation of CXCL12.

It must be noted that our findings do not exclude the possibility of additional mechanisms regulating CXCL12 concentration in the BM. For instance, Petit et al. have reported an enhancement of CXCL12 transcription together with a decrease in CXCL12 protein concentration during GCSF-induced mobilization in the mouse (34). Due to the large amount of active proteases cleaving CXCL12 in the BM (in the range of $1-10 \mathrm{mg} / \mathrm{ml}$ in mobilized BM extracellular fluids) (13, 14) precisely when CXCL12 concentration drops and HPCs mobilize, these results together with ours clearly show that the balance between these two antagonistic effects is largely in favor of proteolytic degradation. It can also be argued that CXCL12 may be complexed to the ECM in the $\mathrm{BM}$, protecting it from proteolytic degradation. However, we have evidence that the ECM in the BM is profoundly altered during mobilization (S.K. Nilsson and J.P. Lévesque, unpublished data), probably due to the fact that most ECM proteins and proteoglycans are also substrates of the neutrophil proteases that cleave CXCL12 (35-40). It is therefore unlikely that the ECM is able to protect CXCL12 within the BM from proteolytic attack.

Taken together, our data demonstrate that the release of neutrophil proteases in the $\mathrm{BM}$, as a consequence of administration of GCSF or CY, results in the cleavage of both the CXCR4 receptor on human CD $34^{+}$HPCs and its ligand CXCL12, which is produced by the BM stroma. It is important to note that the proteolytic cleavage of either CXCR4 (Figure 1) (24) or CXCL12 (Figures 4-6) by neutrophil proteases leads to the complete inactivation of the CXCR4/CXCL12 chemotactic pathway. Previous studies have demonstrated that either systemic administration of CXCL12/CXCR4 antagonists (41, 42 ) or the adenovirus-mediated overexpression of CXCL12 in the liver (43) leads to the mobilization of HPCs in the mouse. Therefore, the proteolytic inactivation of CXCR4 expressed by primitive HPCs, combined with the decrease of CXCL12 concentration in the $\mathrm{BM}$, is likely to promote primitive hematopoietic cell mobilization.

Previous studies have shown that perturbation of VLA-4-mediated adhesion to the stromal cellexpressed counter-receptor VCAM-1 is also sufficient to induce mobilization (10-12). Of great relevance to this and to the current studies is our recent reports that the neutrophil proteases that inactivate CXCR4 and CXCL12 also cleave VCAM-1 $(13,14)$, removing an adhesive ligand with a well-documented role in restricting primitive hematopoietic cells to the $\mathrm{BM}$ $(6,9,10,44,45)$. Thus, administration of GCSF or chemotherapy to induce blood stem cell mobilization results in the concomitant cleavage of both an adhesive substratum (VCAM-1) and a chemotactic axis (CXCR4 and CXCL12), which together are critical mediators of the retention of primitive hematopoietic cells in the BM. An important issue that is left unresolved by our data is which of these two events, cleavage of VCAM-1 or disruption of the CXCR4/CXCL12 axis, is necessary for GCSF-induced and GCSF/chemotherapy-induced mobilization of HPCs. Is one event necessary and sufficient or are both required? Given that administration of either anti-VCAM-1 mAbs (12) or CXCR4 antagonists (41) can induce significant increases in the levels of circulating HPCs, this might suggest that disruption of either axis alone is sufficient to elicit mobilization. However, the level of mobilization resulting from either of these two perturbations is relatively low when compared with that typically observed after administration of GCSF, suggesting that although blockade of either axis is sufficient to induce mobilization, neither alone provides an optimal stimulus. A more likely scenario, therefore, is that the combination of these two events triggers the egress of HPCs into the PB. In favor of this notion are data demonstrating a functional interdependence between these two pathways. For example, CXCL12 has been shown to potently modulate the affinity state of both $\beta 1$ and $\beta 2$-integrins on HPCs (17). The relative inefficiency of anti-VCAM-1-induced mobilization might therefore be explained by the maintenance of an intact chemotactic axis activating alternative integrin-dependant adhesive interactions. On the other hand, among all the currently known CC and CXC chemokines, the chemotactic responsiveness of hematopoietic stem cells is uniquely restricted to CXCL12 (46), suggesting a much lower level of redundancy in the chemotactic pathways controlling migration of HPCs. Given the remarkably restricted chemokine responsiveness of HPCs, it is reasonable to speculate that the proteolytic inactivation of the CXCR4/CXCL12 axis may represent an event of greater importance to the phenomenon of mobilization than the cleavage of VCAM-1. Further experiments mimicking the cleavage of VCAM-1, CXCL12, and CXCR4 in vivo - such as inducible homozygous deletions of VCAM-1, CXCR4, and CXCL12 genes will be required to validate this possibility.

\section{Acknowledgments}

This work was supported by grants from the National Health and Medical Research Council of Australia (to J.P. Lévesque and P.J. Simmons) and from The Leo and Jenny Leukaemia and Cancer Foundation of Australia (L.J. Bendall). The authors wish to thank Ali Amara (Institut Pasteur, Paris, France) for kindly providing $\mathrm{mAb} 6 \mathrm{H} 8$ and Ian Clark-Lewis (University of British Columbia, Vancouver, Canada) for providing synthetic human CXCL12 $\alpha$. 
1. Osawa, M., Hanada, K., Hamada, H., and Nakauchi, H. 1996. Long-term lymphohematopoietic reconstitution by a single CD34-low/negative hematopoietic stem cell. Science. 273:242-245.

2. To, L.B., Haylock, D.N., Kimber, R.J., and Juttner, C.A. 1984. High levels of circulating haemopoietic stem cells in very early remission from acute non-lymphoblastic leukaemia and their collection and cryopreservation. Br. J. Haematol. 58:399-410.

3. Sato, N., et al. 1994. A time course study for optimal harvest of peripheral blood progenitor cells by granulocyte colony-stimulating factor in healthy volunteers. Exp. Hematol. 22:973-978.

4. To, L.B., Haylock, D.N., Simmons, P.J., and Juttner, C.A. 1997. The biology and clinical uses of blood stem cells. Blood. 89:2233-2258.

5. Simmons, P.J., et al. 1994. The mobilization of primitive hemopoietic progenitors into the peripheral blood. Stem Cells. 12:187-201.

6. Arroyo, A.G., et al. 2000. In vivo roles of integrins during leukocyte development and traffic: insights from the analysis of mice chimeric for $\alpha 5$, $\alpha v$, and $\alpha 4$ integrins. J. Immunol. 165:4667-4675.

7. Frenette, P.S., Subbarao, S., Mazo, I.B., von Andrian, U.H., and Wagner, D.D. 1998. Endothelial selectins and vascular cell adhesion molecule-1 promote hematopoietic progenitor homing to bone marrow. Proc. Natl. Acad. Sci. USA. 95:14423-14428.

8. Verfaillie, C.M. 1998. Adhesion receptors as regulators of the hematopoietic process. Blood. 92:2609-2612.

9. Arroyo, A.G., Yang, J.T., Rayburn, H., and Hynes, R.O. 1999. $\alpha 4$ integrins regulate the proliferation/differentiation balance of multilineage hematopoietic progenitors in vivo. Immunity. 11:555-566.

10. Vermeulen, M., et al. 1998. Role of adhesion molecules in the homing and mobilization of murine hematopoietic stem and progenitor cells. Blood. 92:894-900

11. Papayannopoulou, T., and Nakamoto, B. 1993. Peripheralization of hemopoietic progenitors in primates treated with anti-VLA4 integrin. Proc. Natl. Acad. Sci. USA. 90:9374-9378.

12. Kikuta, T., et al. 2000. Mobilization of hematopoietic primitive and committed progenitor cells into blood in mice by anti-vascular adhesion molecule-1 antibody alone or in combination with granulocyte colonystimulating factor. Exp. Hematol. 28:311-317.

13. Lévesque, J.P., Takamatsu, Y., Nilsson, S.K., Haylock, D.N., and Simmons, P.J. 2001. Vascular cell adhesion molecule-1 (CD106) is cleaved by neutrophil proteases in the bone marrow following hematopoietic progenitor cell mobilization by granulocyte colony-stimulating factor. Blood. 98:1289-1297.

14. Lévesque, J.P., et al. 2002. Mobilization by either cyclophosphamide or granulocyte colony-stimulating factor transforms the bone marrow into a highly proteolytic environment. Exp. Hematol. 30:430-439.

15. Aiuti, A., Webb, I.J., Bleul, C., Springer, T., and Gutierrez-Ramos, J.C. 1997. The chemokine SDF-1 is a chemoattractant for human CD34+ hematopoietic progenitor cells and provides a new mechanism to explain the mobilization of $\mathrm{CD} 34^{+}$progenitors to peripheral blood. J. Exp. Med. 185:111-120.

16. Kim, C.H., and Broxmeyer, H.E. 1998. In vitro behavior of hematopoietic progenitor cells under the influence of chemoattractants: stromal cellderived factor-1, steel factor, and the bone marrow environment. Blood. 91:100-110.

17. Peled, A., et al. 2000. The chemokine SDF-1 activates the integrins LFA-1, VLA-4, and VLA- 5 on immature human CD34+ cells: role in transendothelial/stromal migration and engraftment of NOD/SCID mice. Blood. 95:3289-3296

18. Ponomaryov, T., et al. 2000. Induction of the chemokine stromal-derived factor-1 following DNA damage improves human stem cell function. J. Clin. Invest. 106:1331-1339.

19. Ma, Q., et al. 1998. Impaired B-lymphopoiesis, myelopoiesis, and derailed cerebellar neuron migration in CXCR4- and SDF-1-deficient mice. Proc. Natl. Acad. Sci. USA. 95:9448-9453.

20. Zou, Y.R., Kottmann, A.H., Kuroda, M., Taniuchi, I., and Littman, D.R. 1998. Function of the chemokine receptor CXCR4 in haematopoiesis and in cerebellar development. Nature. 393:595-599.

21. Peled, A., et al. 1999. Dependence of human stem cell engraftment and repopulation of NOD/SCID mice on CXCR4. Science. 283:845-848.

22. Jo, D.Y., Rafii, S., Hamada, T., and Moore, M.A. 2000. Chemotaxis of primitive hematopoietic cells in response to stromal cell-derived factor-1. J. Clin. Invest. 105:101-111.

23. Brelot, A., Heveker, N., Montes, M., and Alizon, M. 2000. Identification of residues of CXCR4 critical for human immunodeficiency virus coreceptor and chemokine receptor activities. J. Biol. Chem. 275:23736-23744.
24. Valenzuela-Fernandez, A., et al. 2002. Leukocyte elastase negatively reg ulates stromal cell-derived factor-1 (SDF-1)/CXCR4 binding and functions by amino-terminal processing of SDF-1 and CXCR4. J. Biol. Chem. 277:15677-15689.

25. Takamatsu, Y., et al. 1998. Osteoclast-mediated bone resorption is stimulated during short-term administration of granulocyte colony-stimulating factor but is not responsible for hematopoietic progenitor cell mobilization. Blood. 92:3465-3473.

26. Bendall, L.J., et al. 1997. Antibodies to CD44 enhance adhesion of normal CD $34^{+}$cells and acute myeloblastic but not lymphoblastic leukaemia cells to bone marrow stroma. Br. J. Haematol. 98:828-837.

27. Shen, W., Bendall, L.J., Gottlieb, D.J., and Bradstock, K.F. 2001. The chemokine receptor CXCR4 enhances integrin-mediated in vitro adhesion and facilitates engraftment of leukemic precursor-B cells in the bone marrow. Exp. Hematol. 29:1439-1447.

28. Botos, I., Scapozza, L., Zhang, D., Liotta, L.A., and Meyer, E.F. 1996. Batimastat, a potent matrix metalloproteinase inhibitor, exhibits an unexpected mode of binding. Proc. Natl. Acad. Sci. USA. 93:2749-2754.

29. Eccles, S.A., et al. 1996. Control of lymphatic and hematogenous metastasis of a rat mammary carcinoma by the matrix metalloproteinase inhibitor batimastat (BB-94). Cancer Res. 56:2815-2822.

30. Zimmer, M., et al. 1992. Three human elastase-like genes coordinately expressed in the myelomonocyte lineage are organized as a single genetic locus on 19pter. Proc. Natl. Acad. Sci. USA. 89:8215-8219.

31. Delgado, M.B., et al. 2001. Rapid inactivation of stromal cell-derived factor- 1 by cathepsin $\mathrm{G}$ associated with lymphocytes. Eur. J. Immunol. 31:699-707.

32. Frodl, R., Gierschik, P., and Moepps, B. 1998. Genomic organization and expression of the CXCR4 gene in mouse and man: absence of a splice variant corresponding to mouse CXCR4-B in human tissues. J. Recept. Signal Transduct. Res. 18:321-344.

33. Gupta, S.K., and Pillarisetti, K. 1999. CXCR4-Lo: molecular cloning and functional expression of a novel human CXCR4 splice variant. J. Immunol. 163:2368-2372.

34. Petit, I., et al. 2002. GCSF induces stem cell mobilization by decreasing bone marrow SDF-1 and up-regulating CXCR4. Nat. Immunol. 3:687-694.

35. Janusz, M.J., and Doherty, N.S. 1991. Degradation of cartilage matrix proteoglycan by human neutrophils involves both elastase and cathepsin G. J. Immunol. 146:3922-3928.

36. Kittelberger, R., Neale, T.J., Francky, K.T., Greenhill, N.S., and Gibson, G.J. 1992. Cleavage of type VIII collagen by human neutrophil elastase. Biochim. Biophys. Acta. 1139:295-299.

37. Klebanoff, S.J., Kinsella, M.G., and Wight, T.N. 1993. Degradation of endothelial cell matrix heparan sulfate proteoglycan by elastase and the myeloperoxidase- $\mathrm{H}_{2} \mathrm{O}_{2}$-chloride system. Am. J. Pathol. 143:907-917.

38. Kubes, P., Smith, R., Grisham, M.D., and Granger, D.N. 1993. Neutrophil-mediated proteolysis. Differential roles for cathepsin $\mathrm{G}$ and elastase. Inflammation. 17:321-332.

39. McDonnell, J., et al. 1993. Comparison of the proteoglycanolytic activities of human leukocyte elastase and human cathepsin $\mathrm{G}$ in vitro and in vivo. Connect. Tissue Res. 30:1-9.

40. Steadman, R., et al. 1993. Laminin cleavage by activated human neutrophils yields proteolytic fragments with selective migratory properties. J. Leukoc. Biol. 53:354-365.

41. Shen, H., et al. 2001. CXCR-4 desensitization is associated with tissue localization of hemopoietic progenitor cells. J. Immunol. 166:5027-5033.

42. Broxmeyer, H.E., Hangoc, G., Cooper, S., and Bridger, G. 2001. Interference of the SDF-1/CXCR4 axis in mice with AMD3100 induces rapid high level mobilization of hematopoietic progenitor cells, and AMD3100 acts synergistically with GCSF and MIP-1 $\alpha$ to mobilize progenitors. Blood. 98:3369a. (Abstr.)

43. Hattori, K., et al. 2001. Plasma elevation of stromal cell-derived factor-1 induces mobilization of mature and immature hematopoietic progenitor and stem cells. Blood. 97:3354-3360.

44. Koni, P.A., et al. 2001. Conditional vascular cell adhesion molecule 1 deletion in mice: impaired lymphocyte migration to bone marrow. J. Exp. Med. 193:741-754.

45. Zanjani, E.D., Flake, A.W., Almeida-Porada, G., Tran, N., and Papayannopoulou, T. 1999. Homing of human cells in the fetal sheep model: modulation by antibodies activating or inhibiting very late activation antigen-4-dependent function. Blood. 94:2515-2522.

46. Wright, D.E., Bowman, E.P., Wagers, A.J., Butcher, E.C., and Weissman, I.L. 2002. Hematopoietic stem cells are uniquely selective in their migratory response to chemokines. J. Exp. Med. 195:1145-1154. 